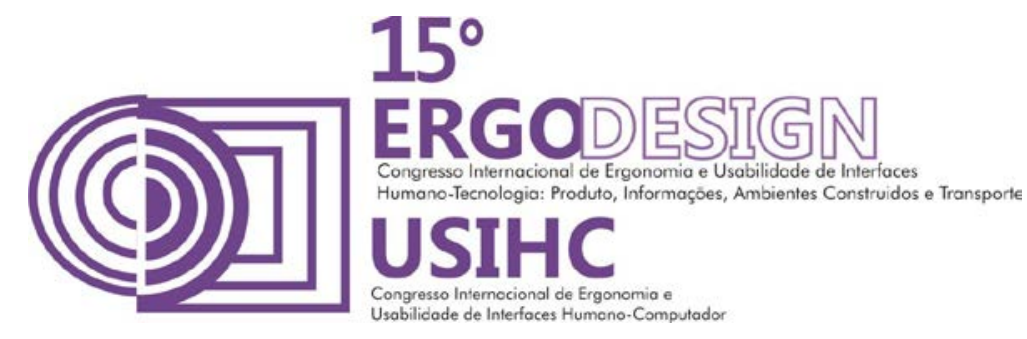

\title{
ANALISE DE CARTILHAS EDUCATIVAS EM SAÚDE: UMA CONTRIBUIÇÃO DA ERGONOMIA INFORAMCIONAL
}

\section{ANALYSIS OF HEALTH EDUCATIONAL BOOKLETS: A CONTRIBUTION OF INFORMATIONAL ERGONOMICS}

\author{
MONT'ALVÃO, Cláudia (1) \\ PEREIRA, Anderson (2) \\ CASSEL, Diana (3) \\ (1) PUC-Rio, Doutora em Engenharia de Transportes . \\ E-mail: cmontalvao@puc-rio.br \\ (2) PUC-Rio, Graduando em Design PUC-Rio \\ E-mail: anderson.vasc.pereira@gmail.com \\ (3) PUC-Rio, Graduanda em Design PUC-Rio \\ E-mail:diana.cassel@gmail.com
}

\begin{abstract}
RESUMO
O presente artigo analisa peças gráficas utilizadas como meio de informação na área da saúde, com o objetivo de contribuir na adequação desses materiais para pacientes diabéticos. No processo da pesquisa foram coletados 21 materiais de educação em saúde e analisados de forma sintática e semântica.

Palavras-chave: Saúde, Cartilhas, Informação, Promoção da Saúde, Educação.
\end{abstract}

\begin{abstract}
The following article analyzes graphic materials used as means of information in health care area. Its principal objective is to contribute to the suitability of these materials for diabetic patients. In the research process, 21 subjects of educational health care area were collected and analyzed syntactically and semantically.

Keywords: Health; Booklets; Information; Health Promotion; Education.
\end{abstract}

\section{CONTEXTO: DOENÇAS EPIDEMICAS E INFORMAÇÃO EM SAUDE}

A Diabetes mellitus tem sido um problema de saúde pública durante anos e crescente na população mundial. De acordo com o dados mostrados na World Diabetes Day 2014 foi visto 387 milhões de pessoas com diabetes no mundo, incluindo um gasto total de 612 bilhões de dólares com a doença. No mesmo ano no Brasil, o número chegou a 1,9 milhões, tendo 


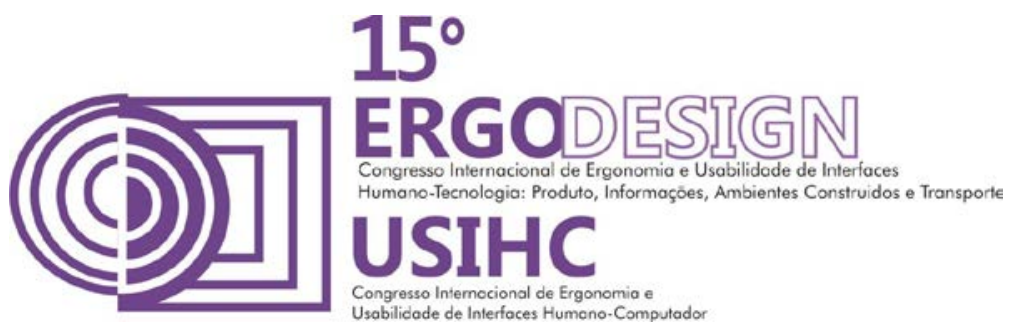

aproximadamente 2,8 milhões de pessoas ainda não diagnosticadas seguido de um custo de aproximadamente 1500 dólares por pessoa. É alarmante sendo o Brasil o país com maior número de diabéticos na América do Sul, levando em conta que $90 \%$ dos diabéticos são do tipo 2 , ou seja, a diabetes que provém dos hábitos do portador em boa parte dos casos. (SBD, 2014).

A Diabetes do tipo 2 é uma doença silenciosa relacionada a problemas cardiovasculares, que costuma se desenvolver geralmente a partir dos 40 anos de idade. A diabetes pode ser evitada se for identificada em um estágio inicial conhecida como Pré-Diabetes, que é apresentada quando um indivíduo possui um nível de glicose entre $100 \mathrm{gm} / \mathrm{dL}$ a $125 \mathrm{gm} / \mathrm{dL}$. Nessa etapa ainda não é considerada diabetes, mas tem uma tendência a evoluir a doença. Ao mesmo tempo é uma fase propícia para prevenir a diabetes do tipo 2, reeducando a pessoa por meio de hábitos alimentares e a prática regular de exercícios físicos. Entretanto só é possível identifica-la através de exames e análise em laboratórios, por não ser comum apresentar sintomas da diabetes. (Souza et al., 2012).

Há muitas informações que cercam a doença por ser complexa e sistêmica e que o paciente deve obrigatoriamente saber para seguir o tratamento. Por esse motivo existem vários meios de comunicação e de auxílio que podem ser utilizados e que também são aplicados pelo governo para a população.

Um deles é a Promoção da Saúde que foi criada a partir da necessidade de melhorias nas condições de vida e assistência médica. De acordo com Buss (2000), graças aos avanços políticos, econômicos e sociais possibilitou o desenvolvimento de tecnologias e contribuições científicas para a saúde pública, além de ainda continuar crescendo ao decorrer dos anos.

Essa promoção é uma estratégia criada para enfrentar problemas de saúdes, sendo normalmente planos e programas elaboradas pelo Governo. Inicialmente o conceito se referia apenas no âmbito da medicina preventiva, porém sua conceitualização mudou a partir das Conferências Internacionais onde se estabeleceram princípios éticos e políticos, principalmente aos valores requisitados pela Carta de Ottawa. (WHO, 1986)

Para o enfoque na saúde, a Carta de Ottawa evidencia a equidade em todos os seus elementos para que haja uma criação de ambientes favoráveis, transformando a saúde em uma responsabilidade global. Contudo, desde a criação da medicina preventiva até a modernização do termo, tem a educação em saúde como um item de grande importância para os objetivos da promoção.

De acordo Buss (2000), baseando no conceito de promoção de Sutherland \& Fulton (1992), instrumentos educativos são destacados quando há a existência de atividades em cima de hábitos e estilos de vida de um individuo, buscando uma relação mais aprofundada e mudanças no comportamento. Desse modo demonstra a relevância da qualidade de um informativo para o individuo aprender a conviver e controlar a sua doença adequadamente.

\section{CONHECIMENTO DOS PERFIS DE DIABÉTICOS JÁ EXISTENTES}

A Ergonomia entende que produtos devem ser adequados aos usuários (Moraes \& Mont'Alvao, 2010), e é a partir dessa perspectiva que dessa pesquisa foi desenvolvida.

A proposta de análise tem o objetivo de verificar cada elemento gráfico e conteúdo do material, mesmo que este não esteja tratando da informação sobre diabetes. Dessa maneira, o importante é observar o modo como as informações sobre saúde estão sendo apresentadas, e se estas estão adequadas aos pacientes. Espera-se que os resultados possam contribuir na elaboração de novas peças informativas. 


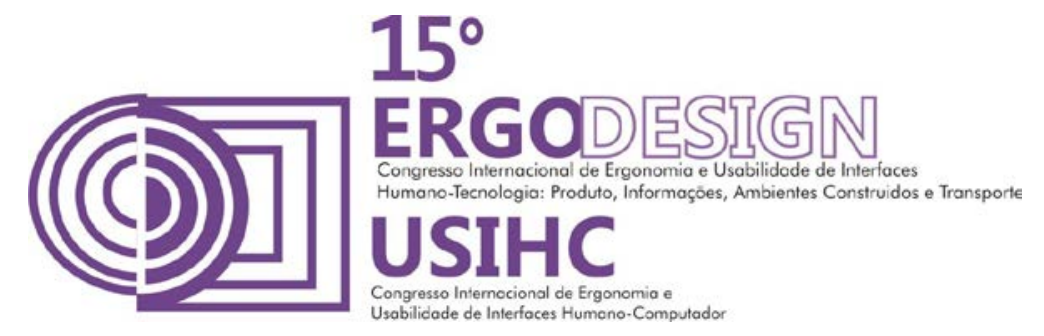

Por isso, antes de proceder a análise gráfica, foi preciso levantar referências que permitissem conhecer o perfil da população diabética. Para isso foi realizada uma busca de artigos que apontassem resultados de campanhas e pesquisas de campo realizadas no Brasil. Essa informação merece destaque, uma vez que a maior parte das referências compilam dados mundiais e/ou da América Latina. Boa parte das referências encontradas apresentam pesquisas realizadas por meio de um estudo observacional descritivo para relatar as características da saúde humana, incluindo um recolhimento dos dados de prontuários e fichas MS HIPERDIA (fichas de recadastramento de diabéticos e/ou hipertensos das unidades de saúde). (Grillo et al., 2007).

Em uma dessas pesquisas, onde o levantamento de dados ocorreu em uma Unidade Básica de Saúde de Porto Alegre, foram apontados como mais relevantes os dados referentes ao nível de escolaridade e a renda dos pesquisados, uma vez que essas variáveis influenciam diretamente na possibilidade de acesso e à aprendizagem do cuidado na saúde. Esse estudo envolveu 206 pessoas, sendo que destas somente 135 participaram efetivamente. Do total dessa amostra, a maioria dos respondentes possui de 1 a 5 anos de estudo, seguido de pessoas não alfabetizadas. (Grillo et al., 2007).

Tabela 1 - Número de pessoas de acordo com a escolaridade em Porto Alegre

\begin{tabular}{|c|c|c|}
\hline & $\mathbf{N}$ & $\%$ \\
\hline Não Alfabetizados & 23 & 18,4 \\
\hline $1-5$ anos de estudos & 81 & 65,6 \\
\hline 6-8 anos de estudos & 11 & 8,8 \\
\hline $7-11$ anos de estudos & 9 & 7,2 \\
\hline
\end{tabular}

Fonte: Grillo et al (2007)

Grillo et al (2007) afirmam que a educação em saúde é uma das principais ferramentas para o autocuidado e a maneira mais indicada para a mudança de estilo de vida, deixando claro que o nível de escolaridade é um desafio para a construção de um material informativo. Os autores também destacam que a renda está diretamente ligada ao grau de instrução, o que pode limitar as condições do paciente tanto no aprendizado, quanto no acesso de alguns serviços de saúde. Dentre os participantes, boa parte recebe de 1 a 2 salários mínimos por mês, enquanto outros vivem na dependência de familiares. 


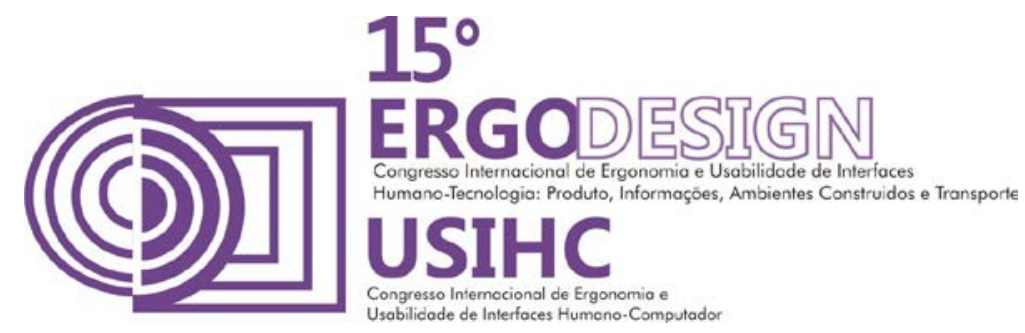

Tabela 2 - Número e pessoas de acordo com a renda mensal

\begin{tabular}{|c|c|c|}
\hline & $\mathbf{N}$ & $\%$ \\
\hline$<1$ salário mínimo & 10 & 8,0 \\
\hline 1 - 2 salários mínimos & 63 & 50,4 \\
\hline $2-3$ salários mínimos & 19 & 15,2 \\
\hline $3-4$ salários mínimos & 2 & 1,6 \\
\hline$>4$ salários mínimos & 8 & 6,4 \\
\hline Não possui renda & 23 & 18,4 \\
\hline
\end{tabular}

Fonte: Grillo et al (2007)

Uma outra pesquisa sobre a prevalência do diabetes realizada pela Universidade Federal de Pernambuco (UFPE) em 2010 (Lyra et al., 2010), também chegou a resultados semelhantes. Dos 198 indivíduos estudados pela Universidade, observou-se que todos os casos de prevalência eram entre pessoas não alfabetizadas ou que tinham apenas o ensino fundamental, além de estarem na faixa de baixa renda. Ambas as pesquisas pontuam os dados socioeconômicos como justificativa no crescimento da prevalência do diabetes. Dessa maneira enfatiza na necessidade de melhorias de estratégias para garantir o acesso de informações à população.

Tabela 3 - Prevalência de Diabetes Mellitus na população de Canaã de acordo com a escolaridade

\begin{tabular}{|c|c|c|}
\hline & $\begin{array}{c}\text { Distribuição Geral da } \\
\text { Amostra (\%) }\end{array}$ & \% de Diabetes Mellitus \\
\hline Analfabetos & 13,1 & 4,30 \\
\hline Ensino Fundamental & 80,0 & 17,1 \\
\hline Ensino Médio & 6,9 & 0,00 \\
\hline
\end{tabular}

Fonte: Lyra et al (2010) 


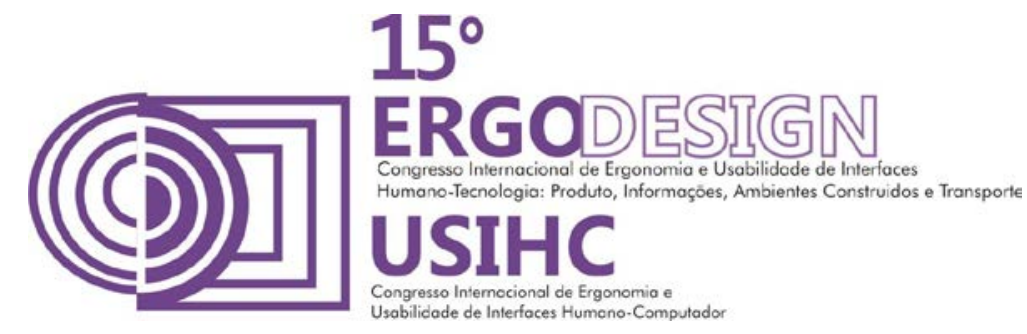

Tabela 4 - Prevalência de Diabetes mellitus na população de Canaã de acordo com a renda

\begin{tabular}{|c|c|c|}
\hline & $\begin{array}{c}\text { Distribuição Geral da } \\
\text { Amostra (\%) }\end{array}$ & \% de Diabetes Mellitus \\
\hline$<1$ salário mínimo & 81,3 & 13,3 \\
\hline 1 - 2 salários mínimos & 16,7 & 10,0 \\
\hline 2 > salários mínimos & 2,0 & 50,0 \\
\hline
\end{tabular}

Fonte: Lyra et al (2010)

Toda a análise de perfil do paciente é importante para a construção de materiais de educação em saúde, por adquirir conhecimento das necessidades dessas pessoas. Em uma terceira pesquisa sobre o processo de construção de material educativo de (Reberte et al., 2012) os autores destacam a vantagem da relação direta com o paciente na elaboração de informativos para gestantes através de resultados positivos no entendimento dos manuais.

De acordo com a pesquisa de (Reberte et al., 2012), o uso de materiais impressos nas unidades básicas de saúde é comum, uma vez que esses materiais tem a capacidade de auxiliar os pacientes, esclarecendo suas duvidas. Portanto, o foco no desenvolvimento de manuais e cartilhas, torna uma forma de contribuir para a Promoção da Saúde.

E ainda, a abordagem da Ergonomia permite que as características, habilidades e capacidades desses usuários sejam consideradas para o projeto adequado desses materiais.

\section{O METODO: A ANÁLISE DE PEÇAS GRÁFICAS}

Inicialmente foram selecionadas um total de 21 peças gráficas, sendo manuais (também chamados de cartilhas), cartazes e histórias em quadrinho disponibilizadas em sites de ONGs, prefeituras estaduais e empresas farmacêuticas. Os materiais tratavam de diabetes, hipertensão, dengue, asma,

A maioria apresentava um apelo visual, sendo que alguns chegavam até a justificar o uso de desenhos para atrair o leitor de forma lúdica. Foi possível classificar essas peças gráficas em: 6 HQs, 9 manuais ilustrados, 5 cartazes ilustrados e apenas um manual sem a utilização de desenhos. Com esse conjunto, procedeu-se uma análise semântica e sintática, em cada um dos seus aspectos. Ao longo desse trabalho somente algumas imagens e aspectos relacionados com a doença foram destacados, para permitir e ilustrar a compreensão do leitor.

\subsection{Quadrinhos (HQs)}

As peças que mais se destacaram graficamente foram as $\mathrm{HQ}$, por serem mais desenvolvidas nos elementos ilustrativos, porém boa parte apresentou problemas em relação à organização dos conteúdos. De acordo com Scott McCloud (2006), a composição deve ter clareza, ser direta 


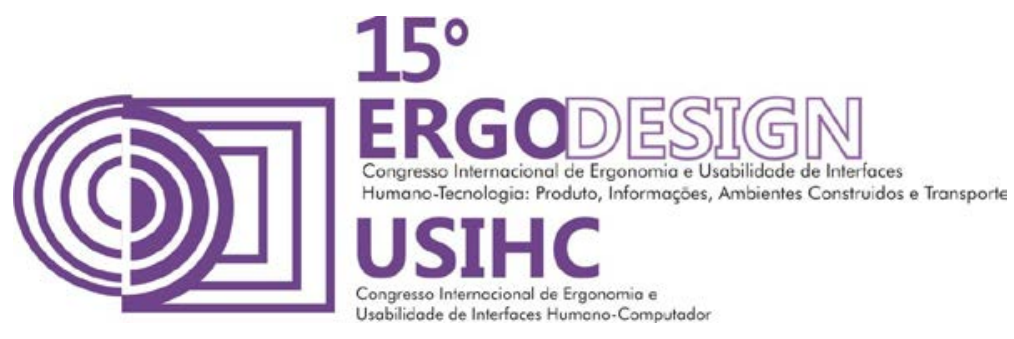

e ter o poder de persuadir o leitor. Por isso, deve existir a preocupação no fluxo e no momento em que cada informação for encaixada e, se for encaixada, dependendo da sua importância. No entanto, dentre os materiais de educação em saúde coletados verifica-se que são carregados informações relevantes sobre o tratamento e cuidados, tais como manutenção de medicamentos, dietas, cuidados para evitar complicações.

McCloud (2006) também afirma que o planejamento do enquadramento favorece a resolução e a organização do fluxo do quadrinho sem comprometer os segmentos dos balões e contribuindo para uma boa leitura. No caso de materiais da área da saúde analisados, como em sua maioria, apresentam conteúdo extenso, entende-se que o ideal seria um fluxo de acontecimentos aumentado para diluir a quantidade da massa de texto presente nos quadros, sem tornar algo cansativo para o leitor.

\subsection{Cartazes e Manuais}

Apesar dos cartazes serem mais usados para divulgação de campanhas do governo, alguns dos cartazes analisados durante a pesquisa também apresentaram o problema de organização do conteúdo. Dois dos três cartazes analisados possuíam informações mais extensas, o que normalmente seria recomendado para outro tipo de material, tal como o manual.

Além dos quadrinhos, os manuais também são outro tipo de material bastante usado para educação em saúde, além de ser o tipo de material mais fácil de ser encontrado. Dos 9 manuais analisados, o Manual de Educação em Diabetes para Pacientes criado pela IEDE (Instituto Estadual de Diabetes e Endocrinologia), foi o que apresentou graves problemas em sua composição gráfica e que será analisado mais detalhadamente nesse item do artigo.

Esse manual foi elaborado por dois médicos da mesma instituição, com a proposta de mostrar informações sobre a doença de forma ilustrativa e de fácil aprendizado para os pacientes. A previsão era que esse manual fosse disponibilizado online no website do IEDE para qualquer pessoa, porém isso não ocorreu (SBEM, 2012).

Desse modo, o Manual de Educação em Diabetes para Pacientes é um volume único, disponível pela biblioteca do IEDE somente para os estudantes da Uni- Rio e médicos da própria instituição, diferente do que havia dito na notícia.

No primeiro capítulo intitulado "1a Aula-Noções Gerais" o Manual introduz como seria a ação da insulina no organismo por meio de dois diagramas que representam a insulina como uma retroescavadeira, podendo induzir o paciente a uma compreensão equivocada da ação da proteína. Isso porque a insulina não tem a capacidade de "carregar" as moléculas de glicose para o interior da célula, ela apenas permite a entrada dessas moléculas. 


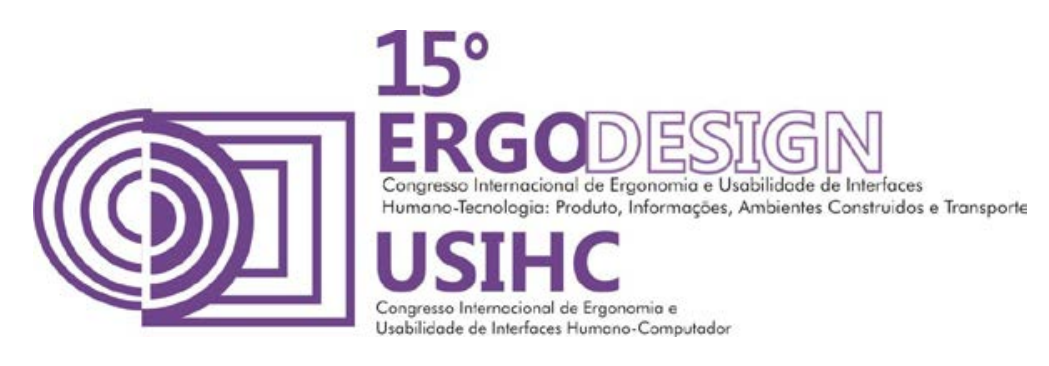

\section{Não diabético}
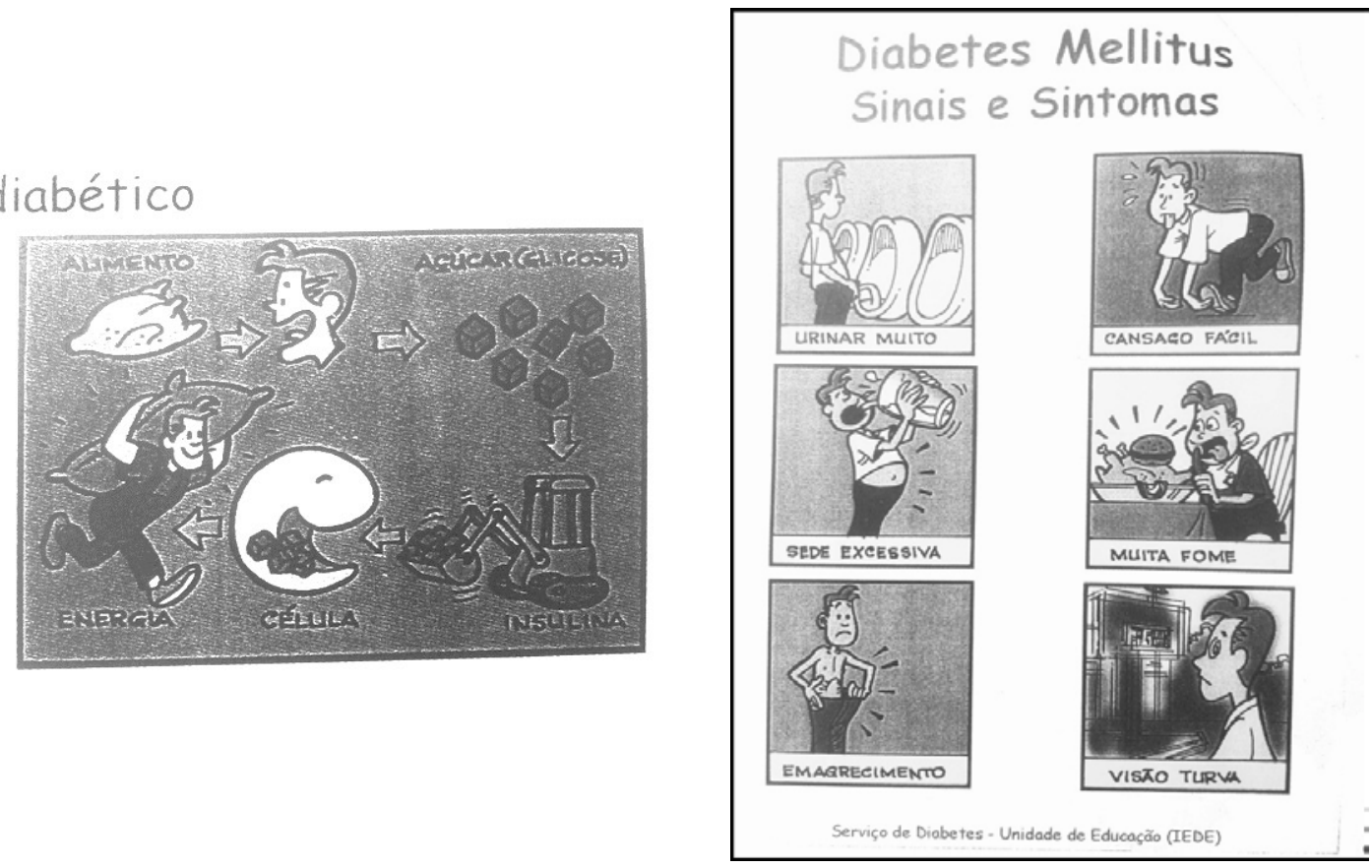
Figuras 1 e 2 - Representação do funcionamento da insulina (à esquerda) e Sinais e Sintomas(à

Fonte: Manual de Educação em Diabetes para Pacientes, IEDE (2012)

Na seção "Diabetes Mellitus sinais e sintomas" os sintomas são expostos de maneira generalizada, sem dividi-los entre as diabetes tipo 1 e 2. O sintoma de emagrecimento é exclusivo do tipo 1 , como também não são comentados alguns sintomas como impotência sexual e demora na cicatrização de feridas.

Algumas informações são dispostas de forma tão sucinta, que comprometem o entendimento da informação. A página 58, p. ex., mostra os tipos de diabetes e alguns tópicos sobre algumas características dos diabéticos, mas não afirma que são as características para um leigo no assunto. Também pode ser considerado um erro colocar o perfil do paciente "Acima de 40 anos" para o diabético tipo 2. A Figura 3 ilustra essa informação. Isso porque segundo a lista de causa de mortes evitais pelo SUS (MALTA et al., 2007) e pela SBD (2014) apresentam dados que demonstram o aumento de casos em pessoas de faixa etária menor.

Além desse fato, existe a possibilidade de gerar um entendimento equivocado dessas faixa etárias, por exemplo, considerar a diabetes do tipo 1 como uma doença que afeta até os 40 anos de idade, ao invés de enxergar como uma doença que deve se manter o cuidado constantemente ao longo da vida. 


\section{(C)] $15_{\text {USIHC }}^{15^{\circ}}$}
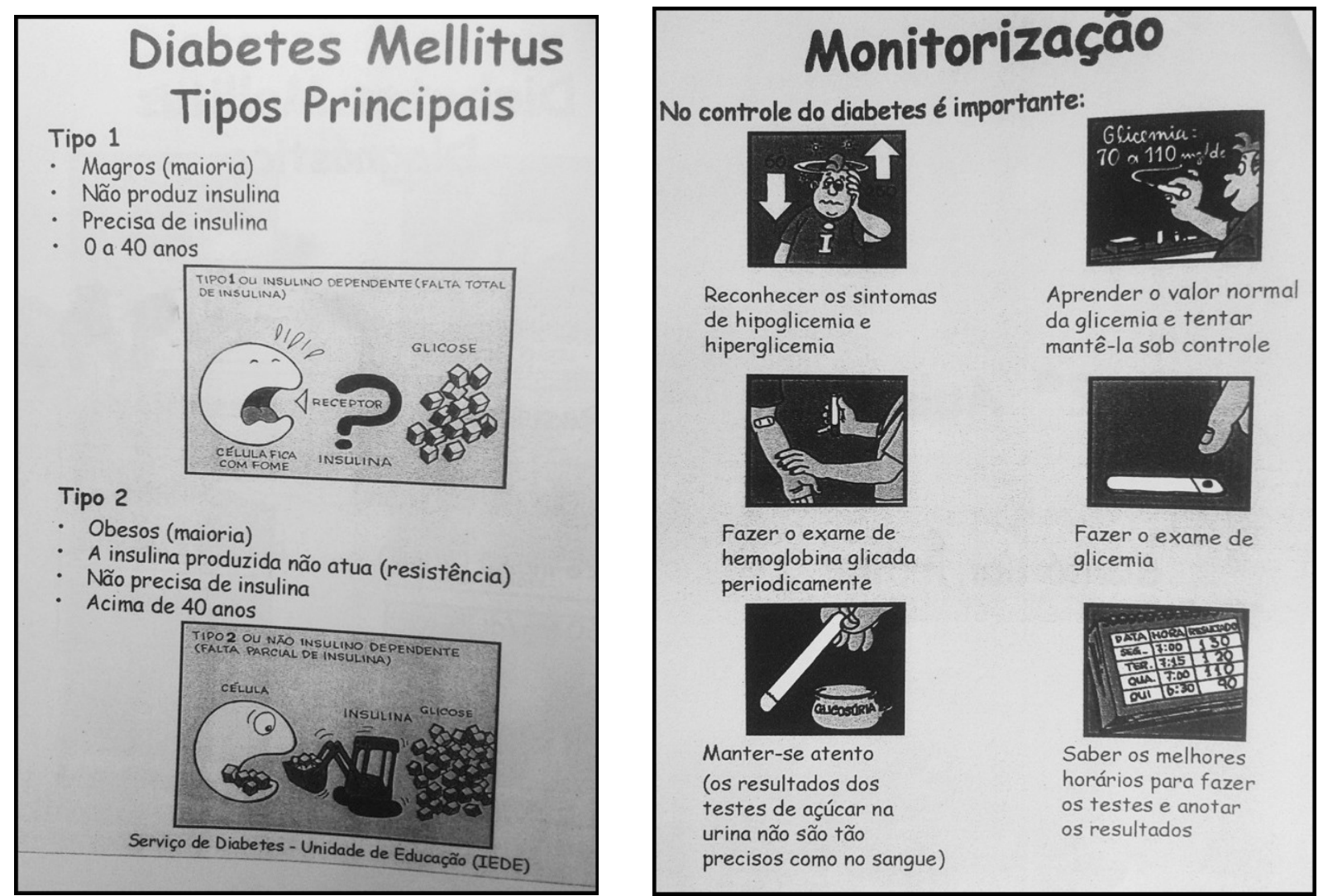

Figuras 3 e 4 - Tópicos sobre tipos de diabetes (à esquerda) e Monitorização (à direita)

Fonte: Manual de Educação em Diabetes para Pacientes, IEDE (2012)

$\mathrm{Na}$ seção "Monitorização", ainda do mesmo manual, e ilustrada pela Figura 5, verifica-se também falta de algumas informações sobre os exames e como reconhecer os sintomas de hipoglicemia e hiperglicemia. Nessa seção, o manual limita-se a avisar que é importante o controle da glicemia e que o paciente deve manter-se atento aos resultados de níveis de açúcar dos exames, sem explicar como são feitos e qual a importância deles.

Logo em seguida, nos complementos do capítulo de monitorização, "Glicemia sem Aparelho" e "Glicemia sem Aparelho", o manual apresenta imagens com uma perspectiva inadequada no modo de usar a caneta lancetador (Figura 5). Essa falha na imagem pode causar uma confusão com a fita usada no glicosimetro. Também no modo de usar, existe a chance do paciente não seguir na sequencia exata, por falta de numeração ou qualquer outro tipo de indicação. Além disso, a cartilha não utiliza o nome glicosimetro, sempre o nomeia apenas como "aparelho". 

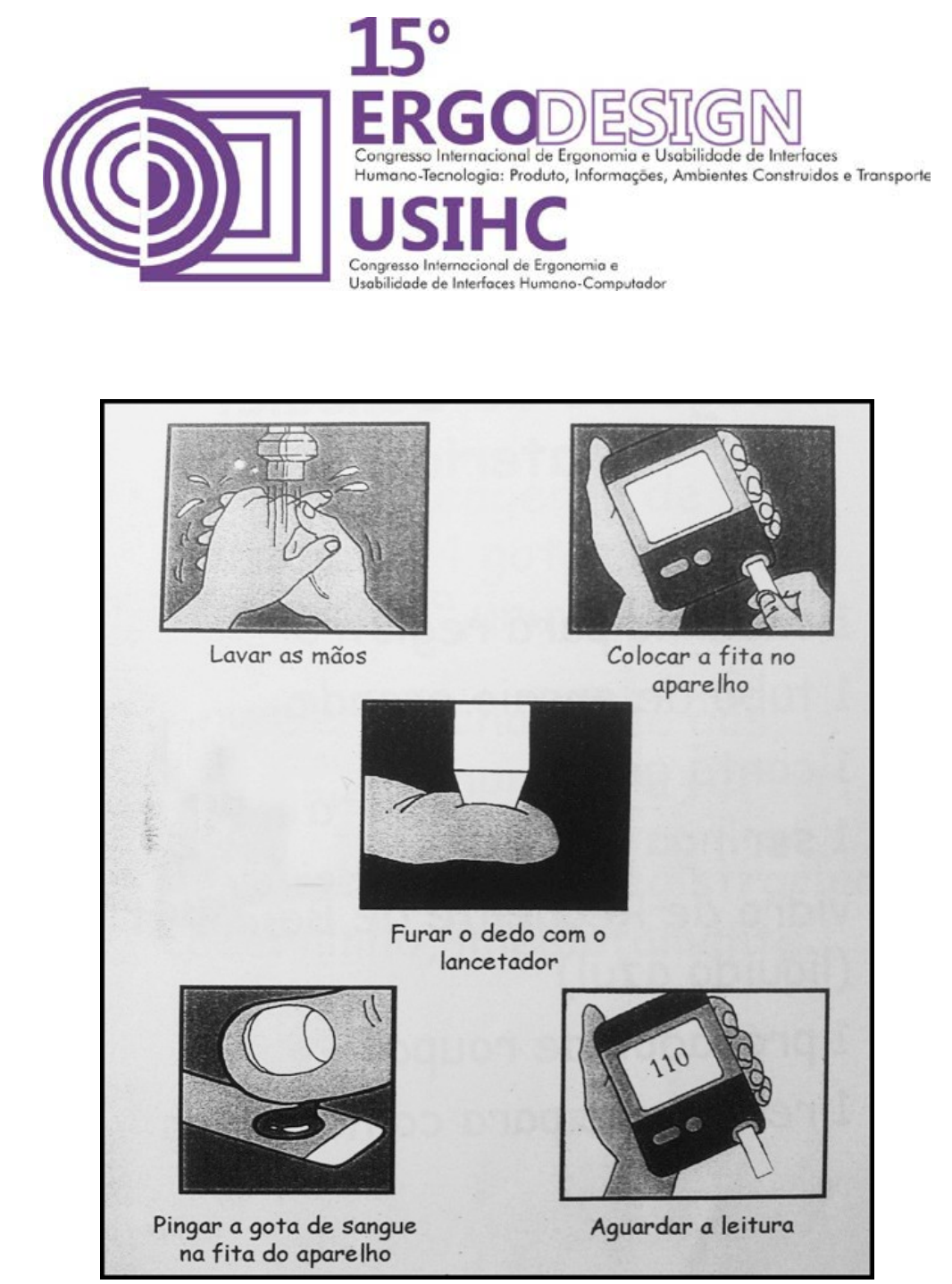

Figura 5 - Modo de usar o lancetador e o glicosimetro Fonte: Manual de Educação em Diabetes para Pacientes, IEDE (2012)

O manual também informa sobre algumas complicações da diabetes, mas quando faz isso apresenta problemas em relação as imagens sobre hiperglicemia (Figura 7), com uma informação superficial, sem considerar alguns sintomas. De acordo com a companhia farmacêutica Lilly (2012), a hiperglicemia pode causar uma frequente perda de peso, tonturas e formigamentos, sintomas que não são informados pelo manual. 

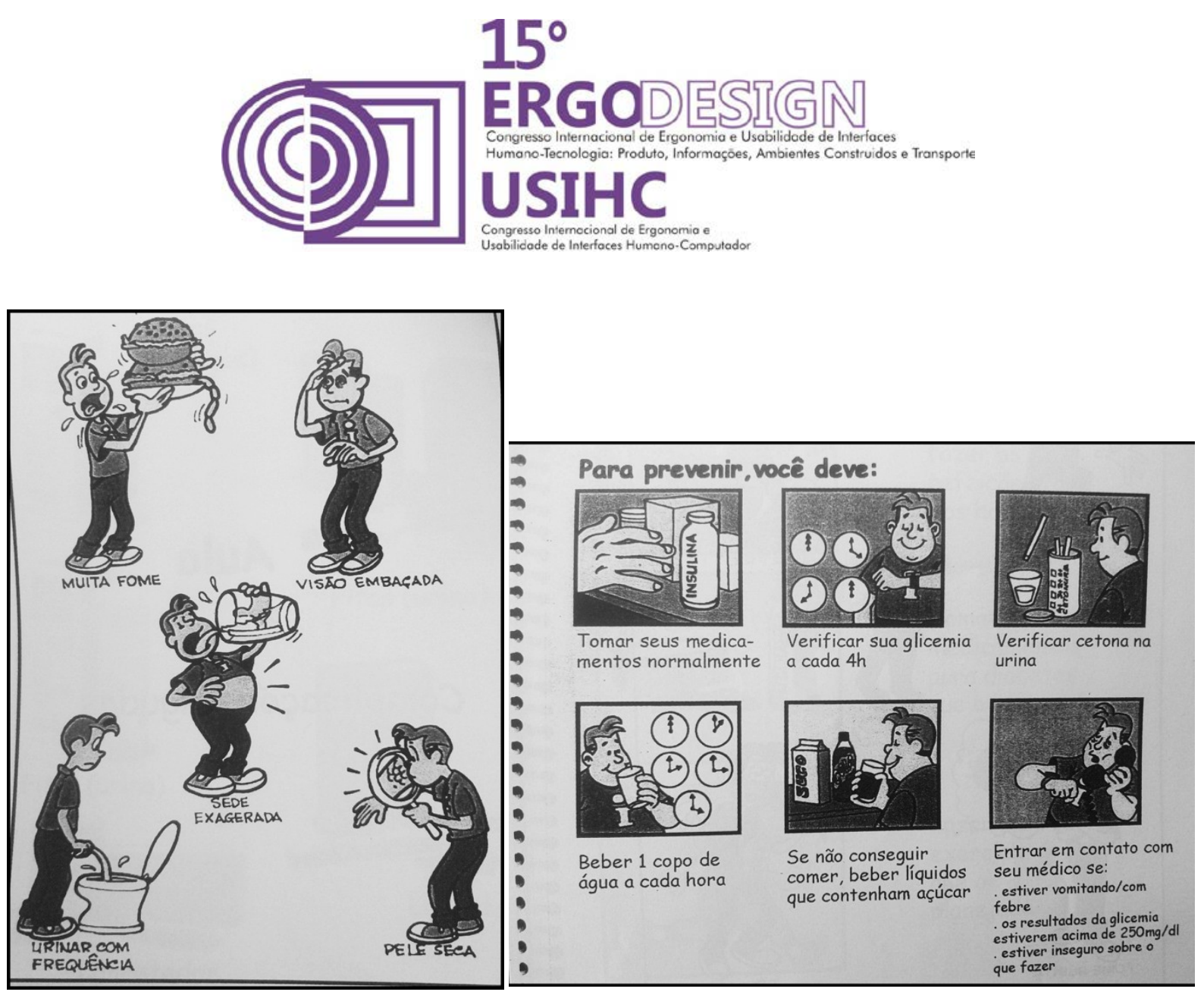

Figuras 6 e 7- Sintomas da hiperglicemia (à esquerda) e Sintomas da glicemia (à direita)

Fonte: Manual de Educação em Diabetes para Pacientes, IEDE (2012)

Ainda que o manual informe sobre sede, fome e vontade de urinar excessiva, não explica o quanto pode ser perigosa essa situação caso o paciente não evite a hiperglicemia imediatamente. Com a elevação de açúcar no sangue e a falta de insulina, as células não têm condições de metabolizar a glicose. Dessa maneira, o corpo reconhece que não há energia suficiente e começa uma produção de um composto cetônico, transformando a gordura em energia e aumentando ainda mais a quantidade de açúcar no corpo. Consequentemente, causa o emagrecimento, o hálito cetônico e a desidratação.

A Figura 8 apresenta as imagens sobre a prevenção da hiperglicemia, mas ainda de maneira sucinta e sem com poucos elementos em como evitar tal situação. Nas formas de prevenção, apresenta um erro grave induzindo o paciente a beber líquidos que contenham açúcar. 


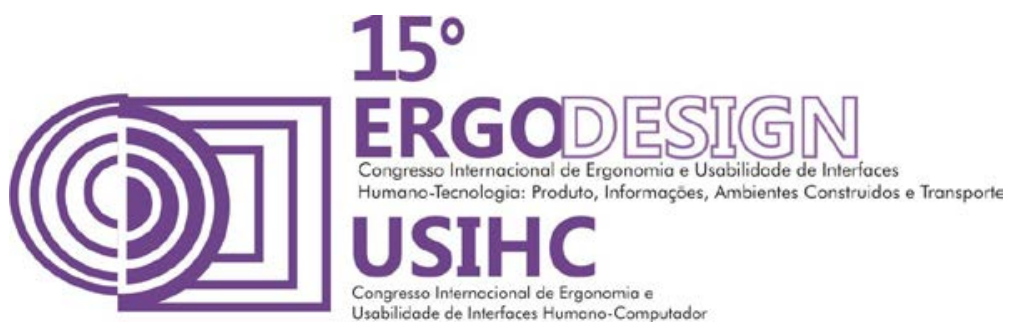

\section{CONCLUSÃO}

A possibilidade de rever os níveis de escolaridade foram essenciais para melhor compreensão do apelo de ilustrações nos conjuntos de peças gráficas. Ainda que se tenha a existência de públicos diferentes, nem todos os materiais analisados foram desenvolvidos de acordo com um perfil de usuários

Algumas cartilhas também não se mostraram adequadas para informar os cuidados com a doença, devido à falta de conteúdos básicos, informações insuficientes, ou em alguns casos, induzindo o paciente ao erro.

Do ponto de vista gráfico, o uso de ilustrações não reconhecíveis, de baixa qualidade ou por apenas como elemento atrativo foram bastante presentes nas maiorias dos materiais analisados, o que requer do paciente/ usuário, elevados níveis de abstração para compreensão da informação.

$\mathrm{Na}$ composição das histórias em quadrinho, foi comum o acúmulo de informações nas páginas, tornando prejudicial ao próprio fluxo. É provável que falta de recursos ou custos elevados para produção desse tipo de material tenha influenciado na elaboração, o que também explicaria a disponibilidade somente na internet da maioria. Contudo, esse tipo de material apresentou uma linguagem obliqua, mais aproximada do público em comparação com os outros tipos de materiais coletados.

Em relação ao manual do IEDE, do qual várias imagens foram apresentadas, esperava-se um material mais próximo do perfil dos pacientes, uma vez que foi elaborado por médicos especialistas no tratamento da doença, mas isso não aconteceu.

Como resultado do trabalho, corrobora-se a necessidade de melhor conhecer o usuário, entender suas características, para que um projeto gráfico possa ser elaborado de forma a proporcionar a esses usuários informação de qualidade, que realmente possa ser utilizado no sentido da Promoção da Saúde. Adequar esses materiais aos usuários é uma contribuição que a Ergonomia e o Design da Informação podem dar aos projetos voltados para Informação em saúde.

\section{REFERÊNCIAS BIBLIOGRÁFICAS}

ALVES, M. J. Q. F.; BICUDO, L.R.H.; KLASSA, B.; GROSSELI, M. M. Gibi educativo: entendendo a hipertensão. Rev. Ciênc. Ext. v.8, n.2, p.113-124, 2012.

Buss, P. M., Promoção da saúde e qualidade de vida, Health promotion and quality of life. Departamento de Administração e Planejamento em Saúde, Escola Nacional de Saúde Pública, Fundação Oswaldo Cruz. Rio de Janeiro, RJ, 2000.

MALTA, D. C., DUARTE, E. C., Lista de causas de mortes evitáveis por intervenções do Sistema Único de Saúde do Brasil. Ministério da Saúde, Secretaria de Vigilância em Saúde, Coordenação-Geral de Doenças e Agravos Não Transmissíveis, Esplanada dos Ministérios, Brasília-DF, 2007.

Grillo MFF, Gorini MIPC. Caracterização de Pessoas com Diabetes Mellitus do Tipo 2. Rev Bras Enferm 2007 jan-fev; 60(1):49-54. 


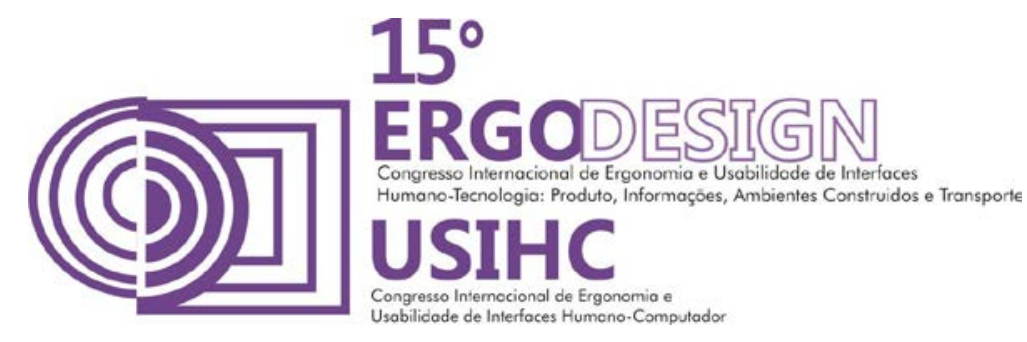

LYRA, R.; SILVA, R.; Prevalência de diabetes melito e fatores associados em população urbana adulta de baixa escolaridade e renda do sertão nordestino brasileiro. Departamento de Genética, Centro de Ciências Biológicas, Universidade Federal de Pernambuco (UFPE), Recife, PE, Brasil, Arq Bras Endocrinol Metab. 2010;54/6

(Moraes \& Mont'Alvao, 2010),

McCloud, S. Desenhando Quadrinhos, Os Segredos e Narrativas de Quadrinhos, Mangás e Graphic Novels. Editora M. Books, 2006

WHO 1986. Carta de Ottawa. Ministério da Saúde/FIOCRUZ. Promoção da Saúde: Cartas de Ottawa, Adelaide, Sundsvall e Santa Fé de Bogotá. Ministério da Saúde/IEC, Brasília.

Anvisa, Guia llustrado, Cartilha sobre Boas Práticas para Serviço e Alimentação. Disponível na internet por http em: < $\underline{\text { http://www.anvisa.gov.br/divulga/public/alimentos/cartilha gicra final.pdf> }}$.

ASSEX IEDE, Diabetes Atualidades, Manual de Educação em Diabetes para Pacientes, Notícia Educação em Diabetes no IEDE, 2012. Disponível na internet por http em: $<$ http://www.assex.org.br/074 diabetes atualidades.asp>. Acesso em 19 de Agosto de 2014

Biblioteca de Livros Digitais, Controla a Asma, Ministério da Educação e Ciência, Governo de Portugal.Disponível na internet por htp $<$ http://www.planonacionaldeleitura.gov.pt/bibliotecadigital/elivro.php?id=controlaaasma $>$. Acesso em 2 de Setembro de 2014

BENFAM, Artigo Enfermagem e Saúde Pública, Acorda Adelaide!!! Disponível na internet por http em: <http://saudepublicaparatodos.blogspot.com.br/2010/01/blog-post.html>.

Educação em Diabetes, História em Quadrinhos Ana e Dinho, Associação Nacional de Assistência ao Diabético. Disponível na internet por http <http://www.anad.org.br/EducacaoDiabetes/quadrinhos.asp>. Acesso em 19 de Agosto de 2014.

Eli Lilly and Company, Vivendo com Diabetes, 2012. Disponível na internet por http em: $<$ https://www.lilly.com.br/Diabetes/Vivendo Com Diabetes/Controlando A Glicemia/Hipoglicemia>. Acesso em 17 de Dezembro de 2014.

Morelli Ortodontia, Gibi Bocaberta. Disponível na internet por http em: $<$ http://www.morelli.com.br/arquivos/gibi/index.htm|\#/6/>. Acesso em 20 de Agosto de 2014.

Portal Prefeitura de Fortaleza, Cartilha da Dengue, Belinha e o Colecionador de Travessuras. Disponível na internet por http em: <http://pt.calameo.com/read/00036052437dc50675a8f>. Acesso em 2 de Setembro de 2014.

Portal da Prefeitura de São, Vigilância em Saúde, Peças Gráficas. Disponível na interne por http em: $<$ http://www.prefeitura.sp.

gov.br/cidade/secretarias/saude/vigilancia em saude/pecas graficas/index.php?p=6755>. Acesso em 2 de Setembro de 2014.

SBD,Sociedade Brasileira de Diabetes, Diabetes já atinge um milhão de crianças no Brasil. Notícia, 2014. Disponível na interne por http em: <http://www.diabetes.org.br/diabetes-na-imprensa/diabetes-ia-atingeum-milhao-de-criancas-no-brasil>. Acesso em 14 de Novembro de 2014.

SBEM, Sociedade Brasileira de Endocrinologia e Metabologia, Manual de Educação em Diabetes para Pacientes, Notícias, 2012. Disponível na internet por http em: <http://www.endocrino.org.br/educacaoem-diabetes/>. Acesso em 19 de Agosto de 2014 